3. Fry WA, Siddiqui A, Pensler JM, Mostafavi H. Thoracoscopic implantation of cancer with a fatal outcome. Ann Thorac Surg 1995;59:42-5.

4. Lewis RJ. VATS is not thoracoscopy. Ann Thorac Surg 1996;62:623-32.

5. Buhr J, Hurtgen M, Kelm C, Schwemmle K. Tumor dissemination after thoracoscopic resection for lung cancer. $J$ Thorac Cardiovasc Surg 1995;110:855-6.

6. Walsh GL, Nesbitt JC. Tumor implants after thoracoscopic resection of a metastatic sarcoma. Ann Thorac Surg 1995;59: 215-6.

7. Lewis RJ, Caccavale RJ, Sisler GE. Does video-assisted thoracic surgery disseminate tumor? J Thorac Cardiovasc Surg 1996;111:1109-10.

$12 / 8 / 82098$

\section{Reply to the Editor:}

We appreciate the comments of Drs. Hermansson, Konstantinov, and Aren in response to our article. Unfortunately, they have misread our conclusion, which may be summarized as follows: The case reports presented raise concerns that the techniques of manipulation of malignant tissue during VATS procedures, regardless of the extent of resection, may lead to an increased rate of suture line occurrences; this increase is the result of inadequate tissue margins caused by an inability to palpate extent of disease. Furthermore, the reported cases raise concerns that disruption of tumorbearing tissue with implantation within the pleural cavity or within chest wall incisions occurs at rates higher than seen during open thoracotomy and intrathoracic resections. The technique they recommend of placing specimens into sealed bags before withdrawal from the chest is reasonable and may spare incisional contamination; however, this will not relieve concerns regarding inadequate margins or disruption within the pleural cavity.

The morbidity and mortality of open procedures for the resection of intrathoracic malignant tumors are well documented in the literature, as is the previously vanishing rare problem of tumor implantation in an incision. Before VATS techniques are generally adopted, we advocate well-designed trials that will document both that VATS techniques are as safe as open techniques and that, stage for stage, VATS techniques provide 3 - and 5-year survivals equal to or better than those achieved with open techniques.

Robert J. Downey, $M D$ Patricia McCormack, MD Division of Thoracic Surgery, Memorial Sloan-Kettering Cancer Center, New York, NY 10021

Joseph LoCicero III, MD Division of Thoracic Surgery New England Deaconess Hospital Boston, MA 02215

12/8/82099

\section{Effect of milrinone on coronary artery bypass grafts}

To the Editor:

The effect of vasodilators on coronary artery bypass grafts is always an interesting topic because the vasoconstriction of the grafts may cause hypoperfusion syndrome, which may cause a serious problem in those who undergo coronary artery bypass with arterial grafts. ${ }^{1}$

With interest, I have read the article authored by Liu and associates ${ }^{2}$ regarding the effect of a relatively new vasodilator, the phosphodiesterase III inhibitor milrinone, which also possesses inotropic effects. In their study, the authors investigated the effect of milrinone in the human internal thoracic artery with regard to the vasorelaxant effect and the role of endothelium in such effect. Their findings in this article are similar to our previous reports in pharmacology journals. ${ }^{3,4}$ In our reports, we have found that milrinone has vasorelaxant effects against all four vasoconstrictors we tested: potassium, U46619, phenylephrine, and endothelin-1. We have also tested the depressant effect of milrinone on the contraction induced by these vasoconstrictors. ${ }^{3}$ In addition, we have found that the vasorelaxant effect of milrinone is endothelium independent. ${ }^{3}$ I am glad to see that these results have been reconfirmed by Liu and his associates. Furthermore, we have discovered that milrinone and nitroglycerin have a synergistic effect that is clinically very important, because these two drugs are sometimes used together in the intensive care unit.

However, on the basis of our findings with regard to the effect and the use of milrinone, we would emphasize three points: (1) Relaxation of milrinone in the potassium-precontracted internal thoracic artery is complete at the highest concentration $(-4 \log \mathrm{M})$, although the sensitivity to this agent is lower (less potent) than to the other three agents. This demonstrates the selectivity of the vasorelaxant effect of this vasodilator. (2) The inhibitory effect of milrinone in the contraction induced by the four important vasoconstrictors is also slightly selective. It was more potent in inhibition of the receptor-mediated (by endothelin-1 and phenylephrine) than the depolarizing agent potassium-mediated contraction (Fig. 1). This is shown by the more significant suppression effect in the contraction induced by endothelin-1 and phenylephrine. ${ }^{3}$ (3) There is a synergistic vasorelaxant effect of the phosphodiesterase III inhibitor milrinone and the nitrovasodilator nitroglycerin in human conduit arteries. This effect may be beneficial to patients undergoing coronary artery bypass grafting and to other patients requiring these vasodilators. Reduced doses of the vasodilators may be sufficient to produce vasodilatation similar to that produced by either of them alone at higher concentrations (Fig. 2). ${ }^{4}$

On the basis of this information, we would suggest clinical considerations and indications for the use of milrinone after coronary artery bypass grafting as follows:

1. The use of milrinone is best indicated when the 